Trinity University

Digital Commons @ Trinity

Psychology Faculty Research

Psychology Department

2004

\title{
A Survey of Psychologists' Attitudes Towards and Utilization of Exposure Therapy for PTSD
}

\author{
Carolyn Becker \\ Trinity University, cbecker@trinity.edu \\ Claudia Zayfert \\ Emily Anderson \\ Trinity University
}

Follow this and additional works at: https://digitalcommons.trinity.edu/psych_faculty

Part of the Psychology Commons

Publication Details

Behaviour Research and Therapy

\section{Repository Citation}

Becker, C.B., Zayfert, C., \& Anderson, E. (2004). A survey of psychologists' attitudes towards and utilization of exposure therapy for PTSD. Behaviour Research and Therapy, 42(3), 277-292. doi: 10.1016/ S0005-7967(03)00138-4

This Post-Print is brought to you for free and open access by the Psychology Department at Digital Commons @ Trinity. It has been accepted for inclusion in Psychology Faculty Research by an authorized administrator of Digital Commons@ Trinity. For more information, please contact jcostanz@trinity.edu. 
Running Head: PSYCHOLOGISTS ATTITUDES TOWARDS EXPOSURE FOR PTSD

\author{
A Survey of Psychologists' Attitudes Towards and \\ Utilization of Exposure Therapy for PTSD \\ Carolyn Black Becker \\ Trinity University \\ Claudia Zayfert \\ Dartmouth Medical School \\ Emily Anderson \\ Trinity University
}

In press: Behaviour Research and Therapy.

Please do not distribute without permission of the authors.

Address correspondence to Carolyn Becker, Ph.D., Department of Psychology, Trinity

University, 715 Stadium Drive, San Antonio, TX 78212-7200. Phone 210-999-8326; Fax 210-

999-8386; Email: carolyn.becker@trinity.edu

Acknowledgments: We are grateful to Brett T. Litz, Kasey Walker, and Christopher Love for their assistance in this study. 


\begin{abstract}
Although research supports the efficacy of exposure therapy for PTSD, some evidence suggests that exposure is under-utilized in general clinical practice. The purpose of this study was to assess licensed psychologists' use of imaginal exposure for PTSD and to investigate perceived barriers to its implementation. A total of 852 psychologists from 3 states were randomly selected and surveyed. An additional 50 members of a trauma special interest group of a national behavior therapy organization were also surveyed. The main survey results indicate that a large majority of licensed doctoral level psychologists do not report use of exposure therapy to treat patients with PTSD. Although approximately half of the main study sample reported that they were at least somewhat familiar with exposure for PTSD, only a small minority used it to treat PTSD in their clinical practice. Even among psychologists with strong interest and training in behavioral treatment for PTSD, exposure therapy is not completely accepted or widely used. Clinicians also appear to perceive a significant number of barriers to implementing exposure.
\end{abstract}

Keywords: PTSD, exposure, cognitive behavioral therapy, survey 
Substantial evidence supports the efficacy of exposure therapy for PTSD (Foa, Keane, \& Friedman, 2000). Yet, as with empirically-supported treatments (ESTs) for many other disorders (Barlow, Levitt, \& Bufka, 1999), exposure appears to be under-utilized in clinical practice (Foy et al., 1996). In one of the few papers to examine the clinical utilization of exposure therapy for PTSD, Foy et al. discussed the low utilization of exposure within VA clinics, which are major centers for PTSD treatment in the United States. Foy et al. cited unpublished data by Fontana, Rosenheck, \& Spencer (1993) indicating that exposure was used to treat fewer than $20 \%$ of 4000 veterans with PTSD and that it was used as the primary treatment in only $1 \%$ of cases. Foy et al. also cited unpublished data (Kagan et al., 1993) indicating that, in a program devoted to delivering exposure therapy for veterans with PTSD, exposure was successfully implemented in only $38 \%$ of cases. The obstacles to implementation in "unsuccessful" cases included psychiatric comorbidity (19\%), task problems (19\%), patient refusal (16\%) and medical contraindications (8\%). Research also suggests that, even among experts, exposure utilization appears to be lower than might be expected. In one of the few other reports of exposure therapy utilization, Litz, Blake, Gerardi and Keane (1990) reported that exposure was used in only 58\% of cases treated by 11 nationally recognized experts in the use of exposure therapy for PTSD.

The limited data on exposure therapy utilization suggest that systematic attention to the myriad of factors that can affect clinical use of exposure is warranted, particularly if advocates of ESTs want to see exposure therapy implemented on a broader scale in general clinical practice. Convincing practitioners to use exposure for PTSD on a routine basis likely will not be an easy task. ESTs such as exposure therapy have been a clinical option for a significant period of time, and it appears that telling practitioners that such treatments exist and that they should use them does not lead to widespread utilization of such interventions. Although the data described by Foy 
et al. (1996) demonstrate that patient barriers can interfere even when therapists are trained and committed to delivering exposure, it is likely that therapist factors play an equally important role in determining whether or not exposure therapy for PTSD is widely utilized.

Several authors have noted that therapists avoid using exposure because they do not understand it and/or are uncomfortable with it (Boudewyns \& Shipley, 1983; Fontana et al., 1993, cited in Rogers, 1998). Dissemination of exposure therapy also hinges on other therapist factors, such as ease, cost, and availability of training; willingness to receive training; availability of supervision; general clinical myths about exposure for PTSD as a whole; and clinical lore and personal beliefs about the specific contexts and conditions for its use. In addition, such therapist variables as knowledge and comfort may interact with a variety of patient variables, such as avoidance or initial worsening of symptoms, to result in low utilization. Finally, professional background and theoretical orientation may play a role in the delivery of ESTs such as exposure therapy. In the U.S, the majority of clinical services are delivered by private mental health practitioners of varying professions, educational experiences, theoretical orientations, and opinions about the role of research in clinical practice. Ultimately, the widespread use of exposure for PTSD likely will depend more upon their attitudes and beliefs about exposure and PTSD than on the opinions of the investigators who develop and test exposure therapy.

In response to calls for increasing dissemination of ESTs, some investigators have begun to examine whether clinicians with limited background in behavior therapy can be trained to effectively deliver exposure therapy (Foa, 2001). This research addresses a very important question, namely can such therapists learn to do this treatment. Other questions, however, remain unanswered, such as whether therapists who can be trained will want to be trained, can be trained within real world financial constraints, and will use the intervention routinely once trained. 
Unfortunately, our impression has been that many clinicians who are trained in exposure do not routinely use it with the majority of their PTSD patients. Thus, in addition to adequate training, other factors such as trust in the intervention, comfort in administering it, and confidence in one's ability to handle a range of patient reactions to it may be vital prerequisites to therapist use of exposure in clinical practice (Litz et al., 1990).

Clinical reports (Jaycox \& Foa, 1996; Pitman et al., 1991) suggest that therapists in clinical practice often encounter barriers that may lead them to discontinue use of exposure therapy with a particular patient, and/or in general. Although some of the obstacles to implementing exposure have been discussed in the literature (Foy et al., 1996; Jaycox \& Foa, 1996; Pitman et al., 1991), data regarding which obstacles most concern clinicians are lacking. Similarly, although data from the VA system have corroborated the perception of low utilization by VA clinicians, comparable data from civilian practitioners are limited, and it is unclear which barriers to implementation pose the most serious problems according to clinicians.

If exposure therapy is to penetrate the clinical realm, researchers and policy makers must understand and address the treatment barriers that are perceived by therapists. As noted by the American Psychological Association Task Force for Empirically Validated Treatments, for clinicians to value research findings may require that clinicians be "treated as partners in the research enterprise" (Task Force on Promotion and Dissemination of Psychological Procedures, 1995, p. 11). Listening to clinician concerns is an important first step to such a partnership. Yet, to our knowledge there have been no published investigations of the clinical utilization of exposure for PTSD in civilian clinical practice. Thus far, practitioner concerns have had to be gleaned from such sources as the non-empirical literature, informal discussions with practitioners who are not research-oriented, informal discussions with scientist practitioners who have left 
exposure for other cognitive behavioral therapy (CBT) approaches, reactions to workshops and presentations on exposure therapy, and the availability of exposure referral options for patients with PTSD. Although such communications have been informative, we were interested to know whether anecdotal impressions would be substantiated by a systematic study of practitioners' reported use of exposure and opinions about factors that influence their decision to use it.

The purpose of the present study was to conduct an initial survey of licensed psychologists regarding their use of exposure therapy for PTSD and their perceived barriers to its implementation. Specifically, we sought to determine the extent to which exposure is being used in clinical practice and to identify factors that may influence psychologists' decision to use it. Thus, we surveyed perceived contraindications to using exposure, expected complications that might arise from its use, and other factors that might influence psychologists' use of it. Because perceptions and use of exposure likely differ between psychologists with defined interest and training in behavioral treatments for PTSD versus clinicians at large, after completing the main survey study we conducted a small follow-up study with a distinct sample of behaviorally trained psychologists interested in PTSD. We hypothesized that only a minority of the main sample of psychologists would report using exposure and that psychologists from this sample would endorse a wide variety of perceived contraindications to and complications arising from exposure for PTSD. We also hypothesized that a majority of behaviorally trained psychologists with a defined interest in PTSD would report using exposure, but that only a minority would report using it with all or almost all of their patients. Finally we hypothesized that participants in this behaviorally trained sample would continue to endorse a significant number of contraindications and complications, although at a lower rate than the main study sample. 


\section{Method}

\section{Participants}

\section{Main Survey Sample}

We surveyed a total of 852 psychologists from two rural New England states and two urban populations in Texas. Fifty percent $(n=543)$ of the licensed psychologists in New Hampshire and Vermont were randomly selected from listings of doctoral level licensed psychologists provided by these states' licensing boards, and 50\% $(n=309)$ of the licensed and provisionally licensed psychologists in San Antonio and Austin were randomly selected from the listings provided by the Texas state licensing board. The survey packet contained individually addressed cover letters, surveys, and addressed, stamped return envelopes. Psychologists were asked to return the surveys within two and a half weeks upon receipt of the survey packet.

Approximately 200 surveys were mailed every two weeks beginning in November 1999. After all of the surveys had been mailed, we distributed follow up letters to the psychologists thanking those who had returned the surveys and asking for those who had not yet returned the surveys to please do so. Return envelopes were destroyed upon receipt to ensure confidentiality and anonymity. Fifteen Texas surveys and 43 New England surveys were undeliverable, and 115 Texas (39.1\%) and 102 New England (20.4\%) surveys were returned, yielding an overall response rate of $27.3 \%$ after undeliverable surveys were subtracted. This response rate is roughly comparable to other surveys of licensed psychologists (e.g., 30\% in Addis \& Krasnow, 2000).

\section{Behaviorally Trained Sample}

In addition to the main survey, we surveyed a population that we expected would have greater familiarity and training in the use of exposure therapy for PTSD. Subsequent to the mailing of the main study, we requested permission from the president of the Disaster and 
Trauma Special Interest Group (D\&T SIG) of the Association for Advancement of Behavior Therapy (AABT) to survey its members (hereafter referred to as the D\&T SIG sample). After permission was granted, we surveyed all members of the D\&T SIG $(n=50)$ in the same manner as described above. Because many SIG members had a preference for email communication, however, an email copy of the survey, along with the standard cover letter, also was sent to the D\&T SIG list-serve with permission of the list-master. The list-serve email noted that members should have received the survey in the mail and that they could answer either via email or anonymous hard copy. The email also informed SIG members that upon receipt of email responses, we would delete their name, print out an anonymous hard copy and then delete their email. This methodology was used to insure as much anonymity as possible with emailed surveys. Five surveys were returned to sender. We created a temporary list of these five names with the intention of crossing the names off the list if we received an email message from any of these members. Because no emailed surveys were received from these members, we counted them as undeliverable. Twenty-nine surveys were returned, yielding a response rate of $64.4 \%$. Survey Questionnaire

In addition to investigating rates of exposure utilization, we were interested in identifying perceived barriers to the clinical use of exposure. Thus, we completed an exhaustive literature search regarding reported difficulties implementing exposure for PTSD and any warnings about using exposure with certain types of PTSD patients. After compiling a list of potential barriers, we developed a 15-item, 3-page, pencil and paper survey designed to assess the following: participants' familiarity with, training in, comfort with, and actual use of imaginal exposure (IE) for PTSD, training in and utilization of in vivo exposure, use of other trauma-focused treatments for PTSD, characteristics thought to contraindicate the use of IE for PTSD, perceptions regarding 
the likelihood of patient problems being exacerbated by exposure, and factors that influence decisions to use exposure. In order to minimize confusion regarding the exposure questions and because IE is typically considered to be the crucial component of exposure therapy for PTSD, the survey focused on IE. Questions about usage of in vivo and other trauma-focused treatment were included in order to gain a sense of respondents' general background with trauma-focused interventions. We also assessed characteristics of the respondents, including theoretical orientation, primary professional setting, patient hours per week, and number of PTSD patients treated. Although closed-ended response categories for each question were devised to facilitate data analysis, where appropriate, we included space for additional comments.

This study was approved by the Trinity University Institutional Review Board. The survey was constructed in an iterative process. First, with the assistance of an undergraduate student, the first and third authors developed the survey and submitted it to the second author for input. After revision the survey was reviewed by a) a nationally recognized PTSD expert and then b) a colleague with extensive experience in survey design. After completing revisions based on their feedback, we administered pilot surveys to eight licensed psychologists and one postdoctoral fellow. Four pilot respondents had expertise in CBT and IE for PTSD. The remaining respondents were not trained in CBT and did not have PTSD expertise. In addition to completing the survey, pilot participants were asked to provide comments. The final set of revisions was completed based on both the responses and feedback of the nine pilot participants. Results

Sample Characteristics

Main Sample 
A total of 217 (27.3\%) surveys were returned. Ten surveys were unusable as the respondents had completed only the demographic questions. These 10 respondents consisted of 4 school psychologists, 2 industrial organization psychologists, 3 retired psychologists, one psychologist who no longer actively practiced, and one psychologist who had "no opinions on this issue." Thus, the final sample for this study consisted of 207 usable surveys.

The majority of respondents $(58 \%)$ endorsed private practice as their primary professional setting. Combined, less than $20 \%$ worked in medical centers and university settings. The mean number of face-to-face patient hours per week was $18.25(S D=11.28)$. The four most common self-described theoretical orientations were eclectic (37\%), psychodynamic/ analytic (28\%), behavioral or cognitive-behavioral (CBT; 21.3\%), and cognitive (9\%).

\section{$D \& T S I G$}

A total of $29(64 \%)$ D\&T SIG members responded to the survey. In contrast to the main sample, the majority D\&T SIG members endorsed CBT as their primary theoretical orientation $(76 \%, n=22)$. One member endorsed humanistic and the remainder endorsed eclectic as their primary theoretical orientation. Also in contrast to the main sample, only a minority $(10 \%, n=3)$ endorsed private practice as their primary professional setting. Forty-one percent $(n=12)$ and $31 \%(n=9)$ endorsed medical centers and universities respectively as their primary professional setting. The mean number of face-to-face patient hours per week was $9.9(S D=9.1)$. IE for PTSD: Experience, Training, Familiarity, Comfort, and Use

Table 1 shows the number of PTSD patients treated by respondents, the number of respondents trained in IE, the different settings in which respondents received IE training, and the number of respondents trained in in vivo exposure for PTSD and/or in exposure for other anxiety disorders. The majority of the main sample (69\%) had not received any formal training 
in IE for PTSD. In contrast to the main sample, the vast majority of the D\&T SIG sample (93\%) had received formal training in IE for PTSD. The mean number of different types of training in IE was $1.7(S D=1.1)$ in the main sample and $2.7(S D=0.8)$ in the D\&T SIG.

Table 2 shows participants' reported familiarity and comfort with IE. Combined, $47 \%$ of the main sample reported that they were "not at all" or only "slightly" familiar with IE for PTSD. Only 20\% reported being "very familiar." Participants were instructed to endorse their level of comfort with IE only if they were familiar with IE. Of the 207 participants, 85 responded to the comfort question. All participants who reported being very familiar with the IE responded to the comfort question, but the response rate was lower (57\%) among somewhat familiar participants. Five slightly familiar participants also responded to this item. Because it seems reasonable to conclude that participants who did not respond to this item did not feel very comfortable with the treatment, we report percentages based on the total sample. The majority of participants did not report feeling comfortable using IE to treat PTSD, and only 10\% reported feeling very comfortable with IE. In contrast to the main sample, the majority of the D\&T SIG sample (86\%, $n=25)$ reported being very familiar and very comfortable $(72 \%, n=21)$ using IE for PTSD.

Table 3 shows rates of utilization of IE. Consistent with our first hypothesis, only $17 \%$ of respondents in the main sample reported that they currently use IE to treat PTSD. Of the respondents who reported use of IE, $52 \%$, or $9 \%$ of the total main sample, reported using IE with $50 \%$ or more of their PTSD patients. Only $4 \%$ of the total sample reported using IE to treat "all or almost all" of their PTSD patients (i.e., 81\% or more). Among participants who reported using IE, the four most commonly endorsed theoretical orientations were eclectic $(34 \%, n=12), \mathrm{CBT}$ $(29 \%, n=10)$, cognitive $(17 \%, n=6)$, and psychodynamic $(17 \%, n=6)$. 
Results from the D\&T SIG were also consistent with our hypotheses. In contrast to the main sample, a majority (66\%) of the D\&T SIG respondents reported current use of IE to treat PTSD. In addition, $84 \%$ of respondents who reported using IE ( $n=16,55 \%$ of D\&T sample) indicated that they use IE with $50 \%$ or more of their PTSD patients. Yet, only 5 participants (17\%) reported current use of IE with all or almost all PTSD patients (i.e., $81 \%$ or more).

Although fewer main sample participants reported using in vivo exposure $(n=22,11 \%)$ as compared to IE, noticeably more respondents $(n=77,37 \%)$ reported using other traumafocused interventions. Participants who reported using other trauma-focused interventions were asked to note what interventions they utilized. We report answers that were noted by more than 2 respondents. Thirty respondents reported using psychodynamic trauma-focused treatment, 14 reported using other CBT strategies (e.g., cognitive processing therapy, cognitive restructuring), 14 reported using Eye Movement Desensitization Reprocessing therapy (EMDR), and 7 reported using hypnosis. Slightly fewer D\&T SIG participants reported using in vivo exposure $(59 \%, n=$ 17) and other trauma-focused treatments $(55 \%, n=16)$ as compared to IE. Of the respondents in the latter group, 6 reported using cognitive therapy and 5 reported using EMDR.

As noted above, a significant portion of participants in the main sample reported no training in IE. Because one would assume a low rate of utilization among untrained clinicians, we decided to conduct a post-hoc examination of IE use among those trained in IE. Similarly, because PTSD experience might influence participants' use of IE, we also conducted a post-hoc examination of current use of IE among the participants who reported being experienced in the treatment of PTSD (i.e., had treated a significant number of PTSD patients). Table 3 reflects these analyses. Fifty-nine participants reported formal IE training. Respondents trained in IE were significantly more likely to report current IE use as compared to untrained respondents, 
$\chi^{2}(1, N=206)=83.45, p<.001$. Of the trained participants, $54 \%$ reported currently using IE and $29 \%$ reported using it to treat $50 \%$ or more of their PTSD patients. We assume that the 3 respondents who reported using IE but did not report formal training in IE were self-trained. Only $15 \%$ of formally trained respondents reported using IE with $81 \%$ or more of their patients.

Seventy-four respondents reported that they had treated 26 or more patients with PTSD and were classified as very experienced in PTSD treatment. Experienced PTSD clinicians were also significantly more likely to report currently using IE as compared to participants with less PTSD experience, $\chi^{2}(2, N=194)=32.97, p<.001$. Only $3 \%$ of participants who had seen fewer than 10 PTSD cases and 10\% of those who had seen 11-25 cases reported current use. In contrast, $37 \%$ of the more experienced clinicians reported currently using IE to treat PTSD. Only $20 \%$, however, reported using IE with $50 \%$ or more of their PTSD patients.

Perceived Contraindications to the Use of IE

Participants were asked to respond to 20 factors which, based on the PTSD literature, might be viewed as potential contraindications to using IE. These factors included: comorbid psychotic disorder, dissociative identity disorder (DID), bipolar disorder, anxiety disorder, or any comorbid disorder; severe suicidality, dissociation, or depression; any suicidality, dissociation, or depression; physical aggression by patient; physical aggression by other to patient; homicidality; low social support; substance abuse/dependence; severe anger; severe numbing; past adherence problems; and past treatment non-response. Participants rated the likelihood that each factor would contraindicate the use of IE. Thus, participants who believed that a given contraindication was not a problem rated it as very unlikely to contraindicate IE. Respondents who were not at all familiar with IE were instructed to skip this item, and all such main sample respondents did appropriately skip out. Of the 207 participants in the main sample, 105 responded to at least one 
item in this question and comprise the main sample for this analysis. Because of the volume of data produced by this question, we collapsed the "somewhat likely" and "very likely" categories.

Overall, both samples highly endorsed factors that one would expect to be endorsed as contraindicating $\mathrm{IE}^{1}$. For example, factors that are frequently exclusion criteria for randomized clinical trials (RCTs) of PTSD were generally rated as likely contraindications, including: severe suicidality (main sample $=85 \%$, D\&T SIG $=79 \%$ ), comorbid psychotic disorder $($ main sample $=$ $85 \%, \mathrm{D} \& \mathrm{~T}$ SIG $=76 \%$ ) and homicidality (main sample $=81 \%, \mathrm{D} \& \mathrm{~T}$ SIG $=62 \%$ ). Overall, D\&T SIG members endorsed fewer contraindications as likely as compared to the main sample. Consistent with our contraindication hypotheses, the mean number of likely contraindications endorsed by D\&T SIG respondents was $7.5(S D=4.8)$, which was significantly less than the number endorsed by the main sample $(M=11.7, S D=5.3), t(131)=3.88, p<.001$.

Although many expected contraindications were highly endorsed, there were some unexpected findings. For example, a relatively large number of main sample respondents also endorsed such factors as any dissociation (51\%), any comorbid diagnosis (37\%), and a comorbid anxiety disorder (32\%). Yet, surprisingly few respondents in both samples endorsed severe anger (main sample $=54 \%$, D\&T SIG $=21 \%$ ), which empirical research has shown to be a risk factor for poor response to IE (Foa, Riggs, Massie, \& Yarczower, 1995). Similarly, surprisingly few respondents endorsed physical aggression by other to patient (main sample $=52 \%$, D\&T SIG $=$ $35 \%$ ), which is often an exclusion criteria in randomized clinical trials (RCTs) for PTSD.

To examine the influence of such factors as comfort and familiarity with IE on perceived contraindications, we totaled the number of contraindicating factors that each main sample respondent identified as likely. Oneway ANOVAs were used to determine whether the number of perceived likely contraindications was influenced by familiarity and comfort with IE, training 
in IE, experience with PTSD, and theoretical orientation. Because a total of five analyses were performed, a Bonferroni correction was used to adjust the criterion for statistical significance for the ANOVAs to .01 to maintain family-wise error probability at .05 (Kirk, 1968).

Ninety-nine percent of respondents $(n=104)$ endorsed at least one contraindication as likely. There were no significant differences in the number of perceived contraindications based on familiarity with IE, $F(2,102)=2.59, n s$, comfort with IE, $F(3,64)=2.44, n s$, or the number of different ways trained, $F(5,99)=.50, n s$. There was a significant difference in the number of perceived contraindications based on experience in treating PTSD, $F(3,99)=4.10, p<.01$. Posthoc Tukey HSD analyses indicated that participants who reported treating between 26-50 patients with PTSD endorsed significantly fewer contraindications $(M=7.6, S D=5.6)$ than participants who had treated fewer than 10 PTSD patients $(M=12.9, S D=4.5)$ or greater than 50 PTSD patients $(M=12.5, S D=5.7)$. Because IE for PTSD is a behavioral intervention, we also compared the number of perceived contraindications reported by participants who self-identified their orientation as CBT $(M=10.0, S D=5.9)$ to the number reported by all other participants $(M=12.1, S D=5.1)$. There was no significant difference based on orientation, $F(1,103)=3.13, n s$. Results of analyses were essentially unchanged when the number of very likely perceived contraindications was used instead of likely contraindications.

\section{Potential Complications Resulting from the Use of IE}

Participants were asked to respond to 14 potential negative results (complications) that have been discussed in the literature on IE for PTSD. The complications included an increase in re-experiencing, avoidance, or arousal symptoms; dissociation; substance abuse/dependence; overwhelming anxiety; numbing; self injury; suicidality; homicidality; desire to drop out of therapy; physical aggression; relationship problems; and a worsening of the therapeutic alliance. 
Respondents who were not at all familiar with IE were instructed to skip this item, and all such respondents in the main sample did skip out. Of the 207 participants in the main sample, 108 responded to at least one item in this question and comprise the sample for this analysis. As with the previous item, we collapsed the "somewhat likely" and "very likely" categories.

The complications most commonly endorsed as likely were increases in arousal (main sample $=87 \%$, D\&T SIG $=55 \%)$, re-experiencing symptoms (main sample $=83 \%$, D\&T SIG $=$ $62 \%$ ), dissociation (main sample $=76 \%$, D\&T SIG $=41 \%$ ), substance abuse (main sample $=$ $75 \%$, D\&T SIG $=51 \%$, and suicidality $(\text { main sample }=75 \%, 45 \%=\mathrm{D} \& \mathrm{~T} \mathrm{SIG})^{1}$. Concerns about self-injury and drop-out are often mentioned in relation to IE and are likely to be of great concern to clinicians. Sixty-eight percent of the main sample and $48 \%$ of the D\&T SIG reported that they believed that self-injury was likely to be exacerbated by IE, and $59 \%$ and $48 \%$ of the main sample and D\&T SIG respectively believed that IE was likely to increase patients' desire to drop out of therapy. Overall, D\&T SIG members were less inclined than the respondents in the main sample to endorse complications as likely. The mean number of likely complications was $5.8(S D=4.8)$, which was significantly less than the number endorsed by the main sample $(M=9.2, S D=4.0), t(133)=4.25, p<.001$.

As above, we totaled the number of problems each main sample respondent rated as "likely" to be made worse by the use of IE. Oneway ANOVAs employing a Bonferroni correction were used in the main sample to determine the influence of familiarity and comfort, training, experience, and theoretical orientation on perceived complications. There were no significant differences in the number of perceived likely complications based on familiarity, $F(3$, $104)=1.62, n s ;$ comfort, $F(3,68)=3.70, n s ;$ training, $F(5,102)=1.83, n s ;$ PTSD experience, 
$F(3,102)=.17, n s$; or theoretical orientation, $F(1,106)=.06$, $n s$. Results of analyses did not differ when the number of very likely negative outcomes was used instead of likely negative outcomes. Factors Influencing the Decision to Use IE for PTSD

Participants were asked to identify factors that might limit their use of IE by checking items that influenced their decision to use IE. The three most commonly endorsed factors in the main sample were limited training $(60 \%, n=124)$, a preference for individualized treatment over manualized therapy $(25 \%, n=52)$, and concern that the patient would decompensate $(22 \%, n=$ $45)^{1}$. Because IE use was low even among trained participants, we separately examined the data from respondents who reported being trained in IE but who were not currently using IE $(n=27)$. The three most commonly endorsed factors among these respondents were lack of training (40\%, $n=11)$, concern that the patient would decompensate $(35 \%, n=9)$, and a preference for individualized treatment over manualized therapy $(31 \%, n=8)$. D\&T SIG members endorsed fewer factors as compared to the main sample. Only four items were endorsed by more than one of the D\&T SIG members and none were endorsed by even $20 \%$ of the sample.

Because it is possible that limited training is a greater problem for non-behaviorally trained clinicians, we examined the main sample data to see if behaviorally trained clinicians appeared less likely to consider training a limiting factor. Over $50 \%$ of participants in each of the four most commonly endorsed theoretical orientations, including behavioral, reported that lack of training was a limiting factor in their use of IE (eclectic 59\%, $n=45$; psychodynamic $64 \%, n$ $=37$; behavioral/CBT 52\%, $n=23$; cognitive 50\%, $n=9$ ), and the difference between groups was not significant $\chi^{2}(3, N=167)=1.83, n s$.

\section{Discussion}


Proponents of empirically-supported interventions, particularly cognitive behavioral therapies, have increasingly turned from the task of developing and testing interventions to the task of trying to disseminate them to practitioners in the general mental health community. Several authors have discussed the potential barriers to achieving widespread use of empiricallysupported interventions (e.g., Barlow et al., 1999). Studies that investigate factors affecting clinical utilization of empirically-supported interventions have the potential to greatly inform the dissemination process. Such studies (e.g., Mussell et al., 2000) are, however, few in number.

The primary objective of the present study was to investigate psychologists' self-reported rates of utilization of IE for PTSD. The main survey results indicate that a large majority of licensed psychologists in clinical practice are not using IE to treat their PTSD patients. Although approximately half of the main sample reported that they were at least somewhat familiar with IE for PTSD, only a small minority (17\%) used it to treat PTSD. To some extent, the low utilization might be due to lack of training, since less than one third of the main sample reported that they had received formal training in IE. Lack of training was also the most commonly endorsed limiting factor among participants in the main sample. Issues in training in ESTs have been extensively discussed by the Task Force on Promotion and Dissemination of Psychological Procedures (1995). As noted by the Task Force, the typical 3-hour to 3-day workshop is insufficient to introduce a new area of competency. Opportunities for more intensive training and for ongoing supervision are clearly needed if clinicians are to add IE to their clinical repertoire. Yet, only about half of those who had received training reported using IE to treat PTSD, and less than a third of those trained used it to treat at least half of their PTSD patients. This suggests that factors other than training also may be influencing the use of IE for PTSD. 
Higher utilization rates reported by participants who were both trained and experienced in the treatment of PTSD (over $65 \%$ for both samples) suggest that experience with PTSD may interact with IE training. These rates are double that reported by participants who were trained but had less experience. Yet, even among participants who are both trained and experienced with PTSD, utilization remains disappointingly low. Less than half of the trained and experienced participants in the main sample reported using the intervention with $50 \%$ or more of their patients, and almost one third did not use IE at all. Utilization among the D\&T SIG sample was also surprisingly low given that the large majority of this sample had received training and nearly three quarters reported being "very comfortable" with IE for PTSD. Fully one third of this sample indicated that they do not currently use IE and only slightly more than half reported that they use IE with at least half of their patients with PTSD. Moreover, even in this highly trained sample only a small portion (17\%) reported current use of IE with almost all their PTSD patients.

Nearly twice as many main sample participants reported formal training in IE for PTSD $(29 \%)$ as compared to exposure for other anxiety disorders (13\%). Similarly, whereas $93 \%$ of the D\&T SIG respondents reported formal training in IE, only $45 \%$ reported formal training in exposure for other anxiety disorders. Thus, it appears that many psychologists have received specialized training in PTSD exposure without obtaining training in the use of exposure for other anxiety disorders. This was sobering given the wealth of evidence supporting the use of exposure interventions for other anxiety disorders and how long these interventions have been available.

The limited training in exposure for anxiety disorders is problematic for two reasons. First, we believe that gaining experience in the use of exposure for other anxiety disorders can help therapists develop familiarity, comfort, and confidence in the use of exposure generally, before applying it to PTSD (Zayfert, Becker, \& Gillock, 2002). Second, as many as 74\% percent 
of patients seeking treatment for PTSD will evidence a comorbid anxiety disorder (Zayfert, Becker, Unger, \& Shearer, 2002). The extent of generalization of trauma-focused exposure work to comorbid anxiety disorders is not known, and comorbid anxiety disorders often may require focused exposure beyond IE for PTSD (e.g., Becker, 2002). Cognitive-behavioral practitioners who are unfamiliar with methods of delivering exposure for other anxiety disorders may not be prepared to address the range of problems presented by PTSD patients.

Understanding practitioner perceptions of barriers to using IE may inform efforts to promote greater use of IE in clinical practice. Thus, a second aim of this study was to identify perceived barriers and other factors that might influence the decision to use IE. Factors that frequently are exclusions for RCTs of IE (e.g., severe suicidality, homicidality, psychotic disorder) were widely endorsed as contraindications by both samples. The main sample also a) endorsed other factors (any dissociation, any comorbid disorder) which have not been overtly excluded from randomized trials and b) indicated that they believed that IE was likely to lead to an increase in symptoms or problems in therapy such as a worsening in suicidality or substance abuse/dependence. Similarly, more than half of the sample believed that patients would experience an increased desire to drop out of therapy as a result of using IE. The overall high endorsement of such contraindications and complications is disturbing, particularly given the finding that such perceptions did not vary according to training, familiarity, comfort, or theoretical orientation. This suggests that beliefs about contraindications and complications are not limited to those unfamiliar with IE or non-behavioral therapists. Such perceptions likely represent real barriers to the dissemination of exposure for PTSD and need to be addressed.

On the whole, the psychologists in both samples surveyed endorsed many likely contraindications to using IE ( $M=11.7$ for the main sample and 7.5 for D\&T SIG). We view this 
as troubling for two reasons. First, when Frueh, Mirabella and Turner (1995) investigated the effect of implementing the seven contraindications generated by the experts in the Litz et al. (1990) study, they found that $73 \%$ of PTSD patients coming to a VA clinic would be excluded. As Frueh et al. noted, virtually none of the contraindications were empirically based. Frueh et al. encouraged clinicians to not categorically exclude all patients who displayed one or more of the contraindications and to consider that "creative engineering" of exposure might enable the application of IE to a broader population. The results of the present study raise concerns similar to those implied by Frueh et al. Namely, that many patients who could benefit from exposure are inappropriately being excluded based on clinician beliefs that IE is associated with an extensive list of contraindications. It should be recognized that clinicians are likely responding to clinical lore since the list of contraindications used in the present study was generated by the literature on PTSD and trauma focused treatment. Research that establishes which factors truly contraindicate the use of IE is needed. In the meantime, however, teaching clinicians how to "creatively engineer" IE would likely be helpful in reducing the number of perceived contraindications and in potentially changing some of the clinical lore regarding IE and PTSD. In addition, it is important that future research provide clear recommendations for handling "contraindications" and discuss when and whether IE should be used after addressing such obstacles.

The results from this survey suggest that clinicians also may sometimes use IE in the presence of real contraindications that are discussed in the literature and often result in exclusion from RCTs. Negative experiences with using IE may significantly affect future use of IE because use of IE in the presence of contraindicating factors may result in poor outcomes and thereby discourage future use. For example, a surprisingly low percentage of both the main sample and the D\&T SIG endorsed physical aggression by other to patient (53 and 35\% respectively) as a 
contraindication. Current physical abuse is, with good reason, often an exclusionary criterion for admission to RCTs of CBT for PTSD (Foa et al., 1999; Foa, Rothbaum, Riggs, \& Murdock, 1991; Resick, Nishith, Weaver, Astin, \& Feuer, 2002). Ongoing physical aggression presents a significant obstacle for PTSD treatment because the hypervigilance and arousal associated with PTSD may be an adaptive reaction to real danger. The focus of therapy under these conditions should be on establishing current safety rather than decreasing vigilance to actual threats.

Severe anger also was endorsed as a contraindication by a relatively low proportion of respondents in both samples (54 and $21 \%$ respectively). Although severe anger has not been an exclusion criterion in randomized trials, empirical research has shown that anger is a risk factor for poor response to IE (Foa, Riggs, Massie, \& Yarczower, 1995). Supplemental strategies for handling severe anger prior to using IE have been discussed in the literature (Becker \& Zayfert, 2001; Jaycox \& Foa, 1996). On the one hand the anger finding may be viewed as positive, indicating a willingness to use IE once anger has been addressed with supplemental strategies. On the other hand, however, low endorsement of anger as a contraindication might indicate that many clinicians are unfamiliar with the empirical and theoretical literature on IE and anger. If a practitioner uses IE with individuals who evidence severe anger and observes an unfavorable outcome, he or she might abandon the use of IE for PTSD altogether.

Results from the D\&T SIG members indicate that although they perceive a number of contraindications and complications, they do so to a lesser degree than participants in the main sample. This suggests that practitioners with more training and experience with CBT generally, and IE for PTSD specifically, may have more confidence in the broader utility of exposure for ameliorating anxiety and PTSD symptoms. D\&T SIG clinicians differ from the main sample in a number of significant ways, however, in that they have a special interest in PTSD, their 
participation in AABT likely indicates an increased interest in research as compared to clinicians at large, and a greater number are primarily located in universities and medical centers. This suggests that addressing the IE concerns of members of the D\&T SIG likely will not be sufficient to significantly increase the use of IE in the general mental health community because D\&T SIG members did not appear to be representative of psychologists at large.

Despite the differences between the samples, it is important to note that nearly half of the D\&T SIG endorsed an increase in desire to drop out of therapy as likely to occur from using IE for PTSD. Thus, even clinicians with the most training and interest in behavior therapy for PTSD have concerns about how patients react to the use of IE. A noteworthy number of members endorsed a wide range of contraindications and complications, and only a small minority use IE to treat the vast majority of their patients with PTSD. Thus, although it may be insufficient to simply attend to the concerns of the D\&T SIG members, members did express significant concerns which likely need to be addressed.

Several limitations to the present study need to be acknowledged. Although an effort was made to sample from both rural and urban practicing psychologists, the sample was limited to three states, and therefore, the survey may not be representative of the population of practicing psychologists in the U.S. Yet, as with Addis and Krasnow's (2000) large national survey of practicing psychologists, $58 \%$ of our main sample reported that their primary professional setting was private practice (compared to $56 \%$ in Addis and Krasnow). This provides some limited support for the generalizability of the present sample.

The response rate of $27.3 \%$ in the present study was somewhat lower than some other surveys of psychologists (e.g., 55\% in Mussell et al., 2000). D\&T SIG members had a markedly higher response rate than the main sample. Although this might be a result of the email response 
option, it could also indicate that clinicians who are familiar with IE might have been more likely to respond to the survey. Thus, it seems important to recognize that community respondents to this survey may present an overly optimistic picture of exposure utilization. In addition, we only sampled psychologists. Psychologists, due to their scientist-practitioner training, might be more inclined to utilize ESTs than other mental health practitioners. Although the generalizability of these findings to the universe of mental health practitioners may be limited, it is reasonable to assume that IE would be used less by practitioners of other professions.

With regard to rates of exposure utilization, this study provides self-report data of practice behavior, and as such may not accurately reflect actual practice. When we began systematically tracking our own practice behavior, we were surprised to find that we had actually used IE with fewer of our patients than we had intended to (Zayfert \& Becker, 2000). Based on our experience, we expect that self-report of practice rates by psychologists with positive attitudes towards IE may in fact overestimate actual rates of utilization.

In summary, exposure for PTSD appears to be under-utilized in clinical practice. Even among psychologists with strong interest and training in behavioral treatments for PTSD, IE is not completely accepted or widely used. Clinicians appear to perceive a significant number of barriers to implementing exposure. Further research is needed to determine which complications are likely and which contraindications are valid. Nonetheless, clinicians in general practice may not change their perceptions solely based on such research. Greater dialog between clinicians and researchers may validate clinician concerns and open the door to developing and studying creative methods for facilitating the use of exposure therapy for PTSD. 


\section{References}

Addis, M. E., \& Krasnow, A. D. (2000). A national survey of practicing psychologists' attitudes toward psychotherapy treatment manuals. Journal of Consulting and Clinical Psychology, 68(2), 331-339.

Barlow, D. H., Levitt, J. T., \& Bufka, L. F. (1999). The dissemination of empirically supported treatments: A view to the future. Behaviour Research \& Therapy, 37, Suppl(1), S147S162.

Becker, C. B. (2002). Integrated behavioral treatment of comorbid OCD, PTSD, and borderline personality disorder: A case report. Cognitive and Behavioral Practice, 9(2), 100-109.

Becker, C. B., \& Zayfert, C. (2001). Integrating DBT-based techniques and concepts to facilitate exposure treatment for PTSD. Cognitive and Behavioral Practice, 8, 107-122.

Boudewyns, P. A., \& Shipley, R. H. (1983). Flooding and implosive therapy: Direct therapeutic exposure in clinical practice. New York: Plenum.

Foa, E. B. (2001). Successful dissemination of exposure to a community-based clinic. Paper presented at the World Congress of Behavioral and Cognitive Therapies, Vancouver, BC.

Foa, E. B., Dancu, C. V., Hembree, E. A., Jaycox, L. H., Meadows, E. A., \& Street, G. P. (1999). A comparison of exposure therapy, stress inoculation training, and their combination for reducing posttraumatic stress disorder in female assault victims. Journal of Consulting and Clinical Psychology, 67(2), 194-200.

Foa, E. B., Keane, T. M., \& Friedman, M. J. (Eds.). (2000). Practice guidelines from the international society for traumatic stress studies: Effective treatments for PTSD. New York: The Guilford Press. 
Foa, E. B., Riggs, D. S., Massie, E. D., \& Yarczower, M. (1995). The impact of fear activation and anger on the efficacy of exposure treatment for posttraumatic stress disorder. Behavior Therapy, 26(3), 487-499.

Foa, E. B., Rothbaum, B. O., Riggs, D. S., \& Murdock, T. B. (1991). Treatment of posttraumatic stress disorder in rape victims: A comparison between cognitive-behavioral procedures and counseling. Journal of Consulting and Clinical Psychology, 59(5), 715-723.

Fontana, A., Rosenheck, R., \& Spencer, R. A. (1993). The long journey home, III: Third progress report on the specialized PTSD programs. West Haven, CT: Department of Veterans Affairs Northeast Program Evaluation Center.

Foy, D. W., Kagan, B., McDermott, C., Leskin, G., Sipprelle, R. C., \& Paz, G. (1996). Practical parameters in the use of flooding for treating chronic PTSD. Clinical Psychology and Psychotherapy, 3(3), 169-175.

Frueh, B. C., Mirabella, R. F., \& Turner, S. M. (1995). Exposure therapy for combat-related PTSD: Some practical considerations regarding patient exclusion. the Behavior Therapist, 18, 190-191.

Jaycox, L. H., \& Foa, E. B. (1996). Obstacles in implementing exposure therapy for PTSD: Case discussions and practical solutions. Clinical Psychology and Psychotherapy, 3(3), 176184.

Kagan, B. L., Foy, D. W., Haas, B., Reno, R., McDermott, C., Moore, R. G., Hammen, M., \& Leskin, G. A. (1993). Long term effects of flooding on chronic posttraumatic stress disorder symptoms. Paper presented at the International Society for Traumatic Stress Studies, San Antonio, TX. 
Kirk, R. E. (1968). Experimental design: Procedures for the behavioral sciences. Belmont, California: Brooks/Cole Publishing.

Litz, B. T., Blake, D. D., Gerardi, R. G., \& Keane, T. M. (1990). Decision making guidelines for the use of direct therapeutic exposure in the treatment of post-traumatic stress disorder. the Behavior Therapist, 13(4), 91-93.

Mussell, M. P., Crosby, R. D., Crow, S. J., Knopke, A. J., Peterson, C. B., Wonderlich, S. A., \& Mitchell, J. E. (2000). Utilization of empirically supported psychotherapy treatments for individuals with eating disorders: A survey of psychologists. International Journal Eating Disorders, 27, 230-237.

Pitman, R. K., Altman, B., Greenwald, E., Longpre, R. E., Macklin, M. L., Poiré, R. E., \& Steketee, G. S. (1991). Psychiatric complications during flooding therapy for posttraumatic stress disorder. Journal of Clinical Psychiatry, 52(1), 17-20.

Resick, P. A., Nishith, P., Weaver, T. L., Astin, M. C., \& Feuer, C. A. (2002). A comparison of cognitive-procesing therapy with prolonged exposure and a waiting condition for the treatment of chronic posttraumatic stress disorder in female rape victims. Journal of Consulting and Clinical Psychology, 70(4), 867-879.

Rogers, S. (1998). An alternative interpretation of "intensive" PTSD treatment failures. Journal of Traumatic Stress, 11(4), 769-775.

Task Force on Promotion and Dissemination of Psychological Procedures, D. o. C. P. (1995). Training in and dissemination of empirically validated psychological treatments: Report and recommendations. The Clinical Psychologist, 48, 3-23.

Zayfert, C., \& Becker, C. B. (2000). Implementation of empirically supported treatment for PTSD: Obstacles and innovations. the Behavior Therapist, 23(8), 161-168. 
Zayfert, C., Becker, C. B., \& Gillock, K. L. (2002). Managing obstacles to the utilization of exposure therapy with PTSD patients. In L. Vandecreek \& T. L. Jackson (Eds.), Innovations in clinical practice: A sourcebook (Vol. 20, pp. 201-222). Sarasota, Fl: Professional Resource Press.

Zayfert, C., Becker, C. B., Unger, D. L., \& Shearer, D. K. (2002). Comorbid anxiety disorders in civilians seeking treatment for PTSD. Journal of Traumatic Stress, 15(1), 31-38. 


\section{Footnotes}

${ }^{1}$ A table providing a full breakdown of data for this item is available upon request 
Table 1

PTSD and Exposure Experience

\begin{tabular}{|c|c|c|c|c|}
\hline \multirow[b]{2}{*}{ Number of PTSD patients treated } & \multicolumn{2}{|c|}{$\begin{array}{l}\text { Main Sample } \\
\qquad(n=207)\end{array}$} & \multicolumn{2}{|c|}{$\begin{array}{c}\text { D\&T SIG } \\
(n=29)\end{array}$} \\
\hline & $n$ & $\%$ & $n$ & $\%$ \\
\hline Less than 10 & 76 & 36.7 & 6 & 20.6 \\
\hline $11-25$ & 44 & 21.3 & 7 & 24.1 \\
\hline $26-50$ & 25 & 12.1 & 3 & 10.3 \\
\hline $50+$ & 49 & 23.7 & 13 & 44.8 \\
\hline Trained in imaginal exposure & 59 & 28.5 & 27 & 93.1 \\
\hline CE workshop & 27 & 13.0 & 9 & 31.0 \\
\hline Graduate school & 16 & 7.7 & 12 & 41.4 \\
\hline Internship & 14 & 6.7 & 10 & 34.4 \\
\hline Formal postdoctoral fellowship & 7 & 3.4 & 5 & 17.2 \\
\hline Informal postdoctoral fellowship & 10 & 4.8 & 4 & 13.8 \\
\hline Other & 8 & 3.8 & 6 & 20.1 \\
\hline $\begin{array}{l}\text { Trained in in vivo exposure for } \\
\text { PTSD }\end{array}$ & 56 & 27.1 & 22 & 75.9 \\
\hline $\begin{array}{l}\text { Trained in exposure for other } \\
\text { anxiety disorders }\end{array}$ & 26 & 12.5 & 13 & 44.8 \\
\hline
\end{tabular}


Table 2

Familiarity \& Comfort with Imaginal Exposure

\begin{tabular}{lcccc}
\hline & $\begin{array}{c}\text { Main sample } \\
(n=207)\end{array}$ & $\begin{array}{c}\text { D\&T SIG } \\
(n=29)\end{array}$ \\
\hline Familiarity & $n$ & $\%$ & $n$ & $\%$ \\
\hline Not at all & 50 & 24.2 & 1 & 3.4 \\
Slightly & 48 & 23.2 & 0 & 0.0 \\
Somewhat & 67 & 32.4 & 3 & 10.3 \\
Very & 42 & 20.3 & 25 & 86.2 \\
\hline Comfort & & & & \\
\hline Not at all & 149 & 72.0 & 2 & 6.9 \\
Slightly & 16 & 7.7 & 0 & 0.0 \\
Somewhat & 21 & 10.1 & 6 & 20.7 \\
Very & 21 & 10.1 & 21 & 72.4 \\
\hline
\end{tabular}

${ }^{\text {a}}$ This category also includes participants who did not respond to this question but who endorsed lack of familiarity with imaginal exposure. It is assumed that participants who are not familiar with a treatment are not comfortable implementing it. 
Table 3

Utilization of Imaginal Exposure

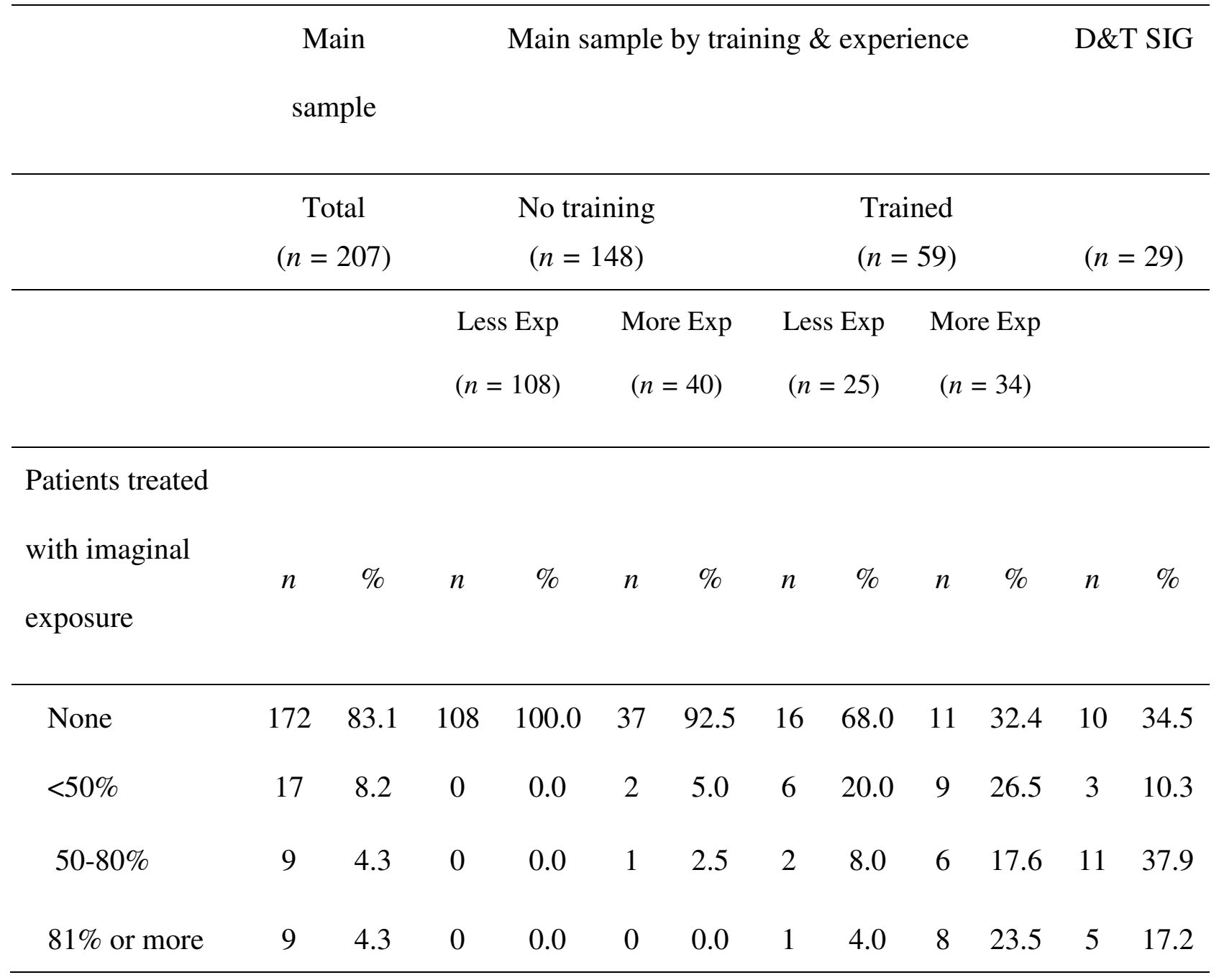

Note. Less Exp = Less Experience, More Exp = More Experience. Clinicians with less experience reported having treating 25 or fewer PTSD patients. More experienced clinicians reported treating 26 or more PTSD patients. 